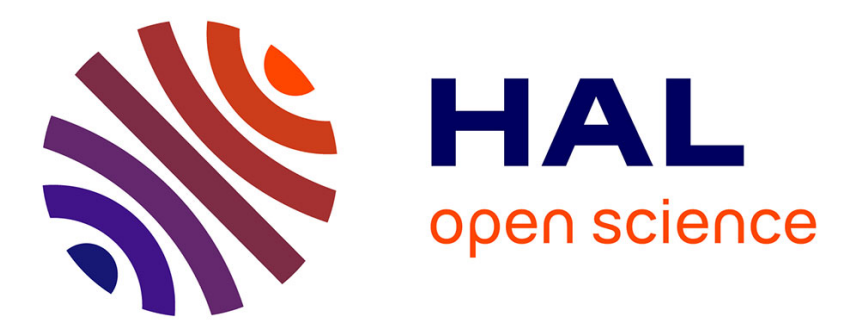

\title{
La dynamique du peuplement et des espaces agraires médiévaux en Berry: propositions pour une évaluation de l'opportunisme des sociétés anciennes
}

Nicolas Poirier

\section{- To cite this version:}

Nicolas Poirier. La dynamique du peuplement et des espaces agraires médiévaux en Berry: propositions pour une évaluation de l'opportunisme des sociétés anciennes. Archéologie médiévale, 2010, 40, pp.15-32. hal-00557193

\section{HAL Id: hal-00557193 \\ https://hal.science/hal-00557193}

Submitted on 13 Jan 2015

HAL is a multi-disciplinary open access archive for the deposit and dissemination of scientific research documents, whether they are published or not. The documents may come from teaching and research institutions in France or abroad, or from public or private research centers.
L'archive ouverte pluridisciplinaire HAL, est destinée au dépôt et à la diffusion de documents scientifiques de niveau recherche, publiés ou non, émanant des établissements d'enseignement et de recherche français ou étrangers, des laboratoires publics ou privés. 


\title{
La dynamique du peuplement et des espaces agraires médiévaux en Berry : propositions pour une évaluation de l'opportunisme des sociétés anciennes
}

\begin{abstract}
Nicolas POIRIER*
Résumé : L'intérêt de mener une étude du peuplement et des espaces agraires dans la longue durée réside notamment dans la possibilité d'apprécier la part d'héritage dont chaque phase de peuplement bénéficie. Les notions d'opportunisme ou d'innovation dans les choix opérés par les sociétés anciennes pour l'implantation des habitats ou des espaces cultivés ne sont que rarement abordées, sans doute en raison de la profondeur chronologique qu'elles nécessitent. Nous proposons ici deux indicateurs d'estimation de l'opportunisme des sociétés médiévales qui, combinés à la restitution du contexte purement environnemental d'installation des habitats et des espaces cultivés, permettent en partie d'expliquer différents épisodes d'emprise, de déprise ou de mobilité du tissu de peuplement et des finages au cours du Moyen Âge.
\end{abstract}

Mots-clés: Berry, peuplement, finage, analyse spatiale, longue durée.

\section{Pour une approche des relations habitat-espace agraire}

\subsection{Une analyse diachronique d'un espace rural à micro-échelle}

Dans le cadre d'une recherche doctorale ${ }^{1}$, nous avons été amené à aborder les dynamiques spatio-temporelles du paysage et du peuplement, au sein d'une microrégion berrichonne, pour tenter de restituer les étapes et les modalités de construction d'un espace rural par les sociétés, de la protohistoire à l'époque contemporaine. L'objectif de cette thèse était de documenter la genèse et l'évolution de chacune des composantes de l'espace rural : des points de peuplement, reliés entre eux par des voies

\footnotetext{
* UMR 6173 CITERES, Laboratoire Archéologie et Territoires, Tours.

1. POIRIER 2010.
} 
de communications irrigant elles-mêmes des territoires d'ordre institutionnel, politique ou de la pratique. Ce travail s'inscrivait donc dans une démarche d'archéologie de l'espace.

La prise en compte de la longue durée était dictée par le constat de l'ancienneté de l'impact de l'Homme sur le milieu et de la rémanence des aménagements du passé dans les formes du paysage actuel ${ }^{2}$. Cette exigence de diachronie est manifeste dans l'étude de la dynamique de l'occupation du sol. La plupart des travaux concernant l'évolution du peuplement sur de courtes périodes chronologiques avouent à un moment du raisonnement le besoin de connaître la situation antérieure pour mieux comprendre la période étudiée $^{3}$. La nécessité de décloisonner les études sur l'occupation du sol est désormais évidente. Ainsi, l'examen des transitions est devenu primordial. Là où des études chronologiquement cloisonnées selon les découpages académiques privilégiaient des scenarii de rupture (entre protohistoire et époque romaine, entre Antiquité et Moyen Âge), les travaux diachroniques renouvellent les schémas de transition en mettant en lumière des phénomènes de continuité ${ }^{4}$. Il faut étudier l'espace et le paysage comme un ensemble dont l'évolution est régie par une temporalité qui lui est propre.

La zone d'étude est située sur la marge orientale du Berry et s'étend sur une cinquantaine de kilomètres carrés, sur le territoire actuel des trois communes de Charentonnay, Sancergues et Saint-Martin-des-Champs (Cher) (fig. 1). L'agglomération villageoise de Sancergues a accueilli au Moyen Âge une communauté de chanoines et le siège d'une châtellenie devenue marquisat à la période moderne ${ }^{5}$.

2. CHOUQuer 1996a ; CHOUQuer 1996b ; CHOUQuer 1997.

3. FAURE-BOUCHARLAT 2001, p. 24.

4. ZADORA-RIO 1987; PARODI 1992.

5. BUHOT DE KERSERS 1892, t.VI, p. 330. 


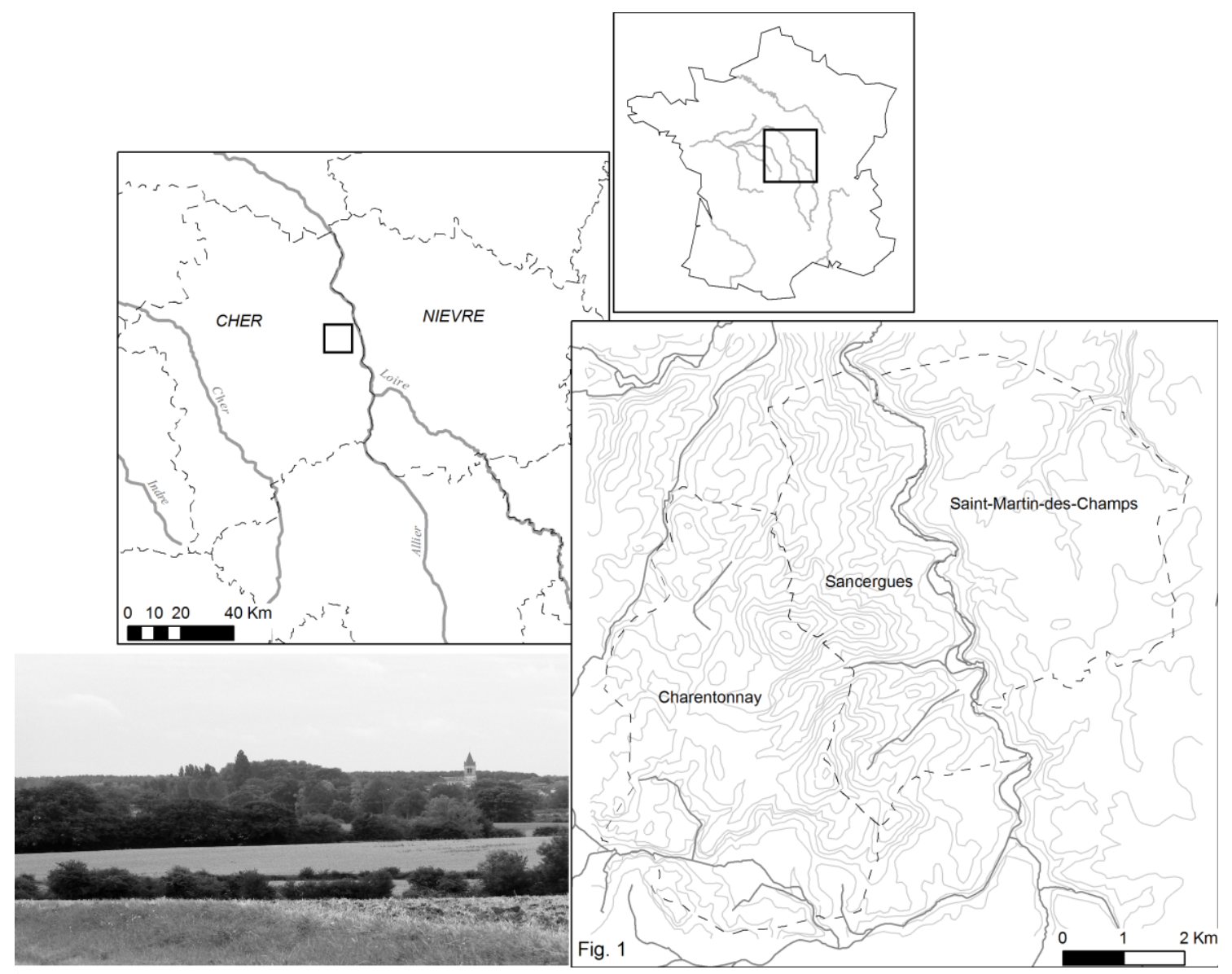

fig. 1 : Localisation de la zone d'étude

Le choix d'un terrain d'investigation spatialement limité était motivé par la volonté d'en faire un laboratoire de réel croisement de données variées (archéologiques, textuelles, planimétriques et environnementales) dans le but de restituer les modalités et les rythmes de construction d'un espace anthropisé par les sociétés successives. De plus, l'objet d'étude étant les relations entre les sociétés successives et leur espace, il fallait bien se placer à l'échelle d'intervention quasi quotidienne des sociétés du passé. Cette échelle est celle des déplacements proches : d'un village à l'autre, du village au hameau, du hameau au champ, du champ à la forêt. L'objet d'étude est le finage, forme d'appropriation de l'espace par la définition de territoires agraires.

\subsection{Des sources d'information variées}

Considérer l'évolution d'un espace depuis la protohistoire jusqu'au XIX ${ }^{\mathrm{e}}$ siècle impliquait la nécessité de traiter plusieurs sources différentes. En effet, si la 
documentation archéologique est la seule disponible pour les périodes les plus anciennes (jusqu'au Moyen Âge), d'autres sources d'informations viennent en complément à partir du $\mathrm{XI}^{\mathrm{e}}$ siècle. Les sources écrites médiévales et modernes et les sources planimétriques (plans terriers, cadastre «napoléonien») fournissent alors un autre point de vue sur l'évolution de l'occupation du sol.

\subsubsection{Les données de prospection au sol}

Nous avons donc développé entre 2003 et 2006 un programme de prospection systématique au sol le long d'un transect documentant la zone d'étude d'est en ouest, traversant ainsi l'ensemble des composantes géomorphologiques du secteur : plateau calcaire de Champagne berrichonne, buttes résiduelles d'argiles à silex, vallée de la Vauvise et limons du val de Loire. L'enjeu était de documenter l'occupation ancienne de ce secteur, jusque-là très mal connu sur le plan archéologique. Les parcelles cultivées ont donc été parcourues par des équipes de quatre à huit personnes, en deux passages successifs dans l'axe des sillons, permettant une détection des sites en observant une maille de cinq mètres et un échantillonnage du mobilier hors-site en observant une maille de dix mètres6. Ces quatre campagnes de prospection ont permis de couvrir plus de 500 ha (soit $10 \%$ de l'ensemble de la zone d'étude), d'identifier une quarantaine de sites d'établissements agricoles dont les occupations s'échelonnent de la protohistoire à la période moderne, mais aussi de documenter l'emprise des espaces cultivés à chaque période par la collecte systématique du mobilier présent hors-site (fig. 2).

6. Procédure en deux passages définie à l'occasion de l'étude la commune de Neuvy-le-Roi en Indre-etLoire (POIROT 1998). 


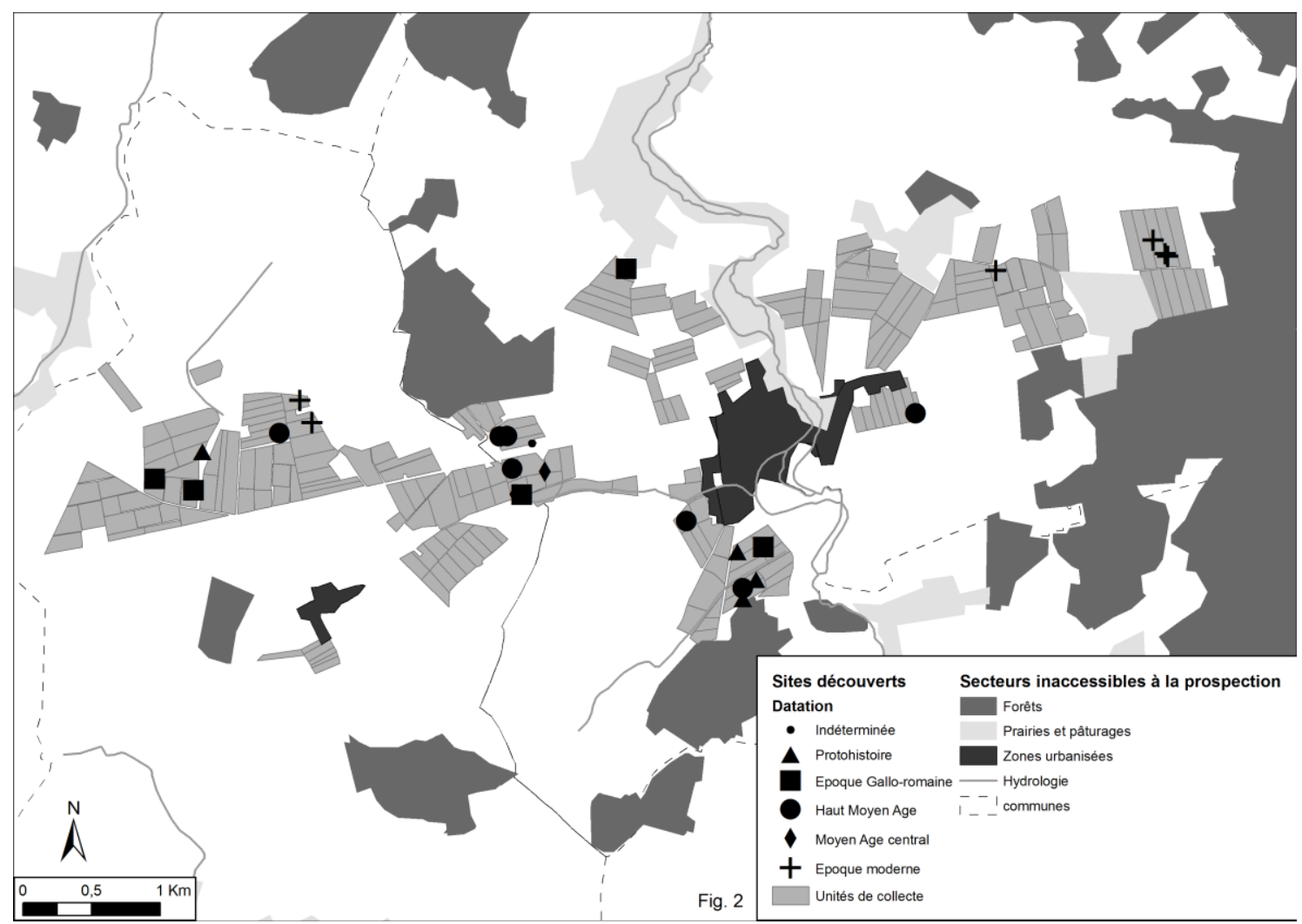

fig. 2 : Carte archéologique à l'issue des prospections

Ce mobilier hors-site, longtemps considéré comme un bruit de fond nuisible à l'identification des sites enfouis, est désormais mobilisé pour restituer l'emprise et l'intensité des amendements en fumures réalisés depuis l'Antiquité, selon le postulat suivant : les tessons de poterie, jetés avec les autres déchets domestiques sur les tas de fumier généralement situés à proximité de l'habitat, ont été épandus avec les matières organiques dans les champs à amender. Une fois les matières organiques décomposées, seuls les éléments solides des fumures subsistent. Les tessons sont donc les seuls témoins de cette pratique agraire et permettent d'analyser l'intensité de l'exploitation de l'espace à l'entour des établissements.

Cette position est défendue depuis les années 1970 par plusieurs chercheurs. On citera en particulier les travaux de G. Foard en Angleterre qui a été l'un des premiers à attirer l'attention sur les vestiges récoltés hors-site ${ }^{7}$. Les recherches menées dans ce sens ont permis de se libérer d'une approche de la prospection systématique centrée sur la découverte des sites, au profit d'une vision continue de l'espace où les vestiges récoltés hors-site livrent une information concernant l'emprise des établissements sur l'espace

7. FOARD 1978. 
environnant. D'autres ont suivi cette voie, surtout des chercheurs anglo-saxons travaillant souvent sur des espaces méditerranéens ou moyen-orientaux ${ }^{8}$. L'intérêt pour le mobilier hors-site (son état de conservation, sa densité et sa répartition) n'est apparu dans un premier temps que pour valider l'existence de sites identifiés, pour faire progresser la procédure de distinction site/hors-site et l'asseoir sur des analyses statistiques, et rarement comme objet d'étude à part entière, à de rares exceptions près 9 . Il faut attendre les années 2000 pour trouver les premiers travaux consacrés à la dynamique des espaces agraires fondés sur l'analyse de la répartition des artefacts récoltés hors-site ${ }^{10}$.

La prudence est néanmoins de mise dans l'interprétation possible des variations que présente la répartition de ce mobilier hors-site dans l'espace et dans le temps. Il ne représente au mieux qu'une estimation minimale des espaces fréquentés et cultivés à chaque pas de temps. En effet, l'absence de vestiges d'amendement agraire ne peut pas être automatiquement considérée comme une preuve d'absence d'occupation. D'autres modes d'utilisation du sol ne laissent aucune trace archéologique (prés et pâturages), tout comme des espaces peuvent être cultivés sans être amendés. Il faut également tenir compte de la circulation et de la consommation différentielle de céramique au cours du temps, ce qui n'est pas possible à l'heure actuelle faute d'un corpus suffisant de sites fouillés dans cette région. Les variations de la quantité de céramique en circulation influencent en grande partie l'image que l'on a de l'intensité de l'exploitation agraire par les seuls épandages de fumures.

Au total, près de 50000 artefacts, dont 7000 tessons de poterie, ont ainsi pu être collectés dans la zone étudiée. L'étude du mobilier céramique a dicté les découpages chronologiques adoptés en fonction de la précision de datation de ce mobilier :

- protohistoire $\left(\mathrm{V}^{\mathrm{e}}\right.$ siècle av. J.-C.-I ${ }^{\mathrm{er}}$ siècle av. J.-C.)

- période romaine $\left(\mathrm{I}^{\mathrm{er}}-\mathrm{III}^{\mathrm{e}}\right.$ siècle $)$

- transition Antiquité tardive-haut Moyen Âge (IV $-\mathrm{VII}^{\mathrm{e}}$ siècle)

- premier Moyen Âge (VIII ${ }^{\mathrm{e}}-\mathrm{X}^{\mathrm{e}}$ siècle)

- $\quad$ second Moyen Âge $\left(\mathrm{XI}^{\mathrm{e}}-\mathrm{XV}^{\mathrm{e}}\right.$ siècle $)$

- période moderne $\left(\mathrm{XVI}^{\mathrm{e}}-\mathrm{XVIII}{ }^{\mathrm{e}}\right.$ siècle)

8. WILKINSON 1982 ; WILKINSON 1989 ; GALLANT 1986 ; BINTLIFF et SNODGRASS 1988.

9. GAFFNEY et GAFFNEY 1988.

10. NUNINGER 2002 ; BERTONCELLO et NUNINGER 2004 ; JONES 2004. 
- période contemporaine $\left(\mathrm{XIX}^{\mathrm{e}}-\mathrm{XX}^{\mathrm{e}}\right.$ siècle $)$

Pour les périodes médiévale et moderne, la datation du mobilier a bénéficié des progrès récents réalisés grâce à la constitution d'une typochronologie régionale dans le cadre d'un Projet collectif de recherches piloté par P. Husi ${ }^{11}$.

\subsubsection{Les sources écrites médiévales et modernes}

Dans le même temps, le dépouillement des sources écrites médiévales et modernes a été réalisé. Deux fonds d'archives principaux documentent la zone étudiée : celui du chapitre canonial Saint-Cyr ${ }^{12}$ et celui de la seigneurie laïque de Sancergues ${ }^{13}$. Ce dépouillement a été mené de manière régressive, privilégiant dans un premier temps les sources sérielles de la période moderne : terriers et censiers. Ces documents, associés à la mobilisation du cadastre napoléonien, ont permis de constituer une importante base de données riche d'environ 900 microtoponymes localisés dans l'espace. Cela a facilité l'exploitation des documents médiévaux, plus rares à mesure que l'on remonte le temps. L'ensemble des sources écrites du $\mathrm{XI}^{\mathrm{e}}$ au XVIII ${ }^{\mathrm{e}}$ siècle a été dépouillé avec le souci constant d'en extraire tous les éléments topographiques, paysagers et territoriaux localisables.

Au total, près d'un millier de mentions de points de peuplements, d'éléments paysagers, du réseau viaire ou de la structure territoriale ancienne ont pu être enregistrés, dont une centaine documentant la seule période médiévale ( $\mathrm{XI}^{\mathrm{e}}-\mathrm{XV}^{\mathrm{e}}$ siècle). La documentation est inexistante avant la fin du XI ${ }^{\mathrm{e}}$ siècle et le nombre de mentions reste faible jusqu'au XIVe siècle, avec seulement quatre éléments enregistrés pour le XII ${ }^{\mathrm{e}}$ siècle, douze éléments pour le XIII ${ }^{\mathrm{e}}$ siècle et dix-neuf éléments pour le XIVe siècle. Un accroissement significatif est notable au XVe siècle (77 éléments), mais la véritable explosion documentaire intervient au XVI ${ }^{\mathrm{e}}$ siècle (355 éléments) confirmée au XVII ${ }^{\mathrm{e}}$ siècle (343 mentions).

Ces différents éléments topographiques localisables ont été enregistrés selon le prisme d'une typologie permettant de distinguer les mentions de lieux habités (actifs ou

11. CNRS - UMR 6173 CITERES, Laboratoire Archéologie et Territoires, Tours - HUSI 2003.

12. AD Cher, sous-série $11 \mathrm{G}$.

13. AD Cher, E 10 à E 167. 
disparus, hameaux, habitats isolés, bâtiments d'exploitation, etc.), les mentions d'éléments du réseau viaire, les mentions d'éléments paysagers (étangs, forêts, nature de l'occupation du sol, etc.) et les mentions de territoires (justices, dîmes, « terroirs », etc.).

Les $\mathrm{XI}^{\mathrm{e}}$, XII ${ }^{\mathrm{e}}$ et $\mathrm{XIII}^{\mathrm{e}}$ siècles ne sont représentés que par des mentions de lieux habités. Aucune information n'est disponible pour ce qui concerne le réseau viaire, le paysage et les territoires de ces périodes (fig. 3).

C'est véritablement à partir du XIVe siècle que sont conservées des mentions documentant le réseau viaire, le paysage et - de façon sporadique - les territoires. À partir de cette date, les mentions textuelles (qu'elles désignent le bâti, le paysage ou les réseaux viaires) sont en progression constante, avec une accélération particulière à partir du XVIe siècle. Il faut toutefois noter l'absence totale d'éléments relatifs aux territoires pour le $\mathrm{XV}^{\mathrm{e}}$ siècle ainsi qu'une légère baisse des mentions concernant le réseau viaire au XVII siècle.

Le dépouillement systématique a été arrêté à la fin du XVII ${ }^{\mathrm{e}}$ siècle dans la mesure où nous disposons pour la première moitié du XVIII ${ }^{\mathrm{e}}$ siècle d'une série de plans terriers documentant une grande partie de la zone étudiée et permettant de faire le lien avec le cadastre napoléonien du début du XIX ${ }^{\mathrm{e}}$ siècle $^{14}$.

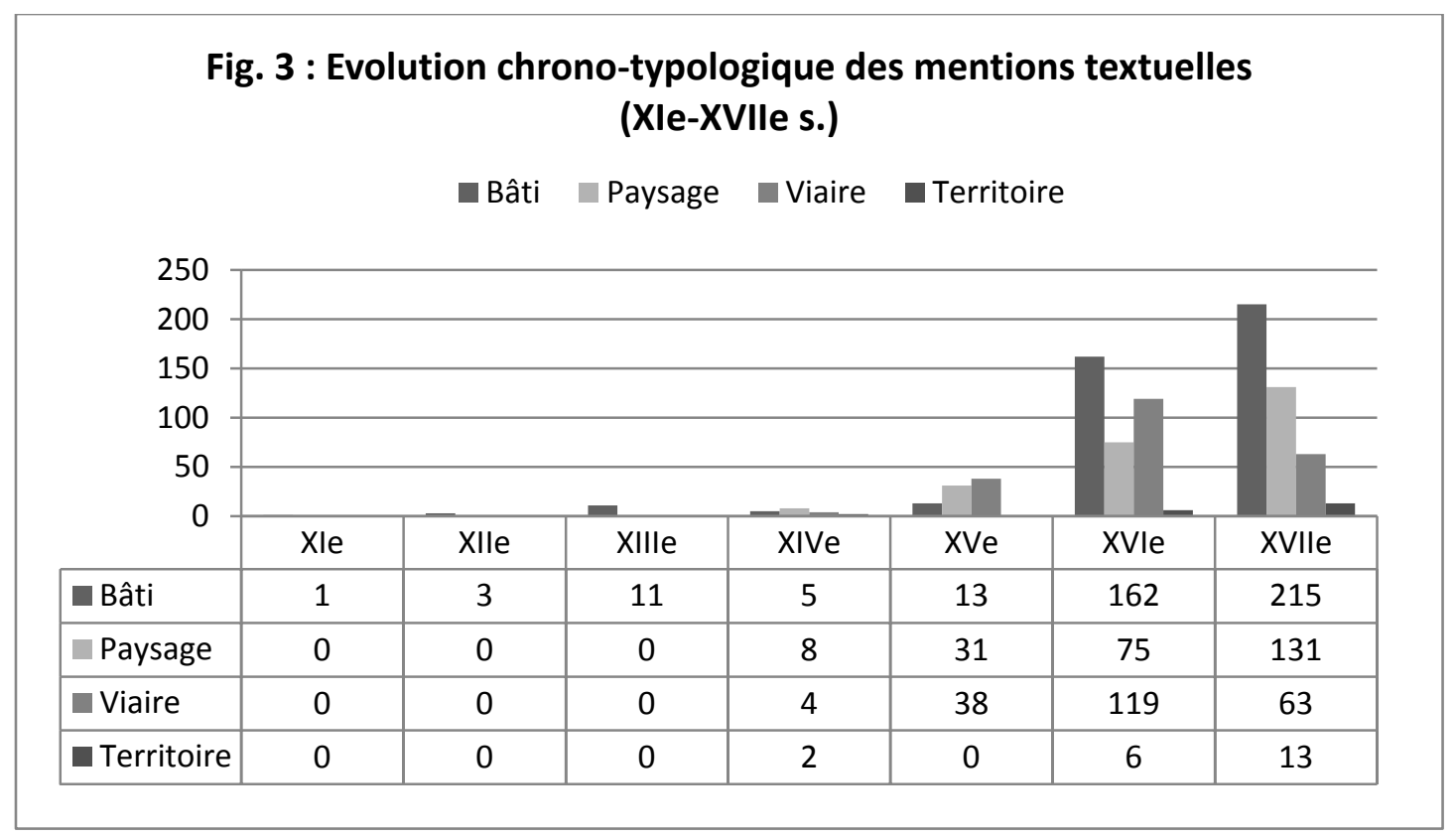

fig. 3 : Évolution typochronologique du nombre de mentions textuelles

14. POIRIER 2006a. 
Archéologie Médiévale, t.40 (2010), p.15-32

\subsection{L'apport d'un SIG : analyses spatiales et statistiques}

La centralisation de l'ensemble de ces informations au sein d'un Système d'Information Géographique a permis le croisement des résultats obtenus à partir de ces différents corpus de sources et ainsi de restituer, l'évolution et les modalités de l'occupation de l'espace. Le recours à la modélisation spatiale sous SIG a notamment permis la mise en évidence des grandes tendances qui affectent l'évolution du paysage dans la longue durée. Cet outil a également favorisé une meilleure appréciation des contextes socio-environnementaux dans lesquels se développent établissements et espaces cultivés, par la confrontation de ces données à différentes couches d'information documentant le relief, le réseau hydrographique ou la qualité des sols.

\subsubsection{Extraire les caractéristiques environnementales des espaces exploités}

L'examen des caractéristiques environnementales (déclivité des pentes, exposition des versants, qualité des sols) est souvent appliqué aux seuls habitats : on interprète alors les résultats en termes de plus ou moins grand confort des habitants en fonction de l'exposition du versant sur lequel est installé le site; on juge de l'adaptabilité des sociétés anciennes en fonction de la déclivité de la pente sur laquelle est bâti l'établissement. Le plus discutable est sans doute d'estimer que le type de sol sur lequel est implantée la ferme est représentatif des types de sols exploités par celle-ci : ce raisonnement est généralement adopté faute de mieux, la plupart du temps à échelle régionale, lorsque l'on ne dispose que du semis des sites occupés à une période donnée, et non des espaces réellement exploités. Cette démarche peut même être biaisée si l'on considère que, plus probablement, les habitats n'ont sans doute pas été implantés à l'endroit exact où les terres étaient intéressantes à cultiver, mais plutôt à leur proximité. L'approche spatiale continue envisagée ici grâce à la prospection au sol et la collecte systématique du mobilier hors-site permet de dépasser ce raisonnement « sito-centré » en cherchant à caractériser les espaces cultivés eux-mêmes, plus probablement sensibles aux aléas climatiques liés à leur contexte (orographique ou pédologique) d'implantation. Ces caractéristiques influent en effet plus sûrement sur la croissance des plantes 
cultivées que sur le degré de confort des paysans.

L'évaluation des critères physiques du milieu repose donc sur l'identification de contextes privilégiés ou délaissés par les sociétés anciennes. On rapporte en effet systématiquement chaque contexte d'implantation à sa représentation dans l'ensemble de la zone étudiée. On considérera par exemple comme ayant été privilégié un type de sol représenté à hauteur de $30 \%$ dans les espaces mis en culture et qui ne constitue que $10 \%$ de la surface totale de la zone d'étude. Cette tendance aura été également vérifiée par les tests statistiques appropriés (test du $\chi^{2}$ d'écart à l'hypothèse d'indépendance).

\subsubsection{Indicateurs de l'opportunisme des sociétés anciennes}

L'appréciation des critères socio-économiques ayant présidé à l'installation de nouveaux établissements, notamment dans la prise en compte de l'héritage des périodes antérieures au Moyen Âge, a nécessité l'invention de nouveaux descripteurs. En effet, les indicateurs généralement utilisés reposent sur l'examen de l'environnement socioéconomique contemporain des sites étudiés : distance par rapport à d'autres installations du même type, par rapport à une agglomération ou à une voie de communication. Ces indicateurs sont difficiles à mettre en œuvre avec des sites découverts lors de prospection, pour lesquels on ne peut pas assurer la contemporanéité de l'activité et qui ne peuvent être datés la plupart du temps qu'à des échelles de temps de trois à quatre siècles.

En revanche, il est possible de mesurer l'opportunisme des sociétés successives en observant les choix d'implantation des établissements en fonction de l'occupation du sol des phases chronologiques antérieures à leur période d'activité. On peut en effet penser que la mise en valeur antérieure d'un espace a pu jouer un rôle dans le choix de tel site plutôt que tel autre.

Deux outils de mesure peuvent être proposés afin d'évaluer d'une part l'influence du tissu de peuplement antérieur (dans quelle mesure ont été privilégiés des sites occupés antérieurement, sur place ou dans un environnement proche), d'autre part l'opportunisme par rapport à la mise en valeur antérieure du terroir. On peut en effet considérer que, la vocation première des établissements identifiés étant probablement l'agriculture, il n'est pas anodin d'implanter le centre de son exploitation au cœur d'un 
espace ayant déjà bénéficié par le passé d'amendements agraires et d'un travail du sol qui a pu contribuer à en améliorer les propriétés.

La prise en compte du peuplement antérieur a d'ailleurs été utilisée dans le cadre du programme Archaeomedes selon une procédure un peu différente de celle proposée ici. Il s'agissait dans ce cas de différencier les établissements dépourvus d'occupation antérieure proche, ceux pour lesquels une occupation antérieure proche était attestée dans le siècle précédent l'installation, et enfin ceux pour lesquels une occupation antérieure proche était attestée plus d'un siècle avant l'implantation considérée ${ }^{15}$. Etant donnée la faible capacité de datation qui ne permet de préciser au siècle près ni l'apparition, ni la disparition des établissements, on propose ici de compter, dans un rayon de $500 \mathrm{~m}$ autour de chacun d'eux, le nombre d'occupations appartenant aux phases chronologiques précédentes. Ce nombre est rapporté au nombre total d'établissements appartenant aux phases chronologiques antérieures à son fonctionnement. On obtient ainsi un indice compris entre 0 et 1 permettant de mesurer l'opportunisme de chaque implantation vis-à-vis du peuplement antérieur (indice de peuplement antérieur).

D'autre part, on peut mesurer l'importance de la mise en valeur agraire de l'espace au sein duquel est implanté un établissement en calculant le nombre de tessons datés des phases chronologiques antérieures à son occupation ayant été récoltés hors site dans les unités de collecte concernées par le rayon de $500 \mathrm{~m}$ alentour. Ce nombre est rapporté au nombre total de tessons datés de ces phases antérieures et récoltés sur l'ensemble du transect prospecté. On obtient ainsi une mesure, en pourcentage, de la part du stock de céramique d'épandage antérieure présente à proximité de chaque établissement et attestant d'une mise en valeur ancienne (indice de mise en valeur agraire antérieure). Cela permet de mesurer l'intensité de la mise en valeur ancienne de l'environnement proche de chaque implantation. Cela complète également l'indice de peuplement antérieur qui ne tient compte que des établissements attestés par l'archéologie à l'exclusion des habitats encore occupés aujourd'hui, puisqu' on ne connaît pas leur date d'implantation. Ce complément est déterminant si l'on considère que l'absence d'habitats antérieurs autour d'un établissement donné ne signifie pas pour

15. VAN DER LEEUW et al. 2003, p. 333. 
autant que celui-ci a été implanté dans un milieu totalement vierge, indemne de toute occupation ou de toute mise en valeur ancienne. La présence d'habitats anciens n'est que la forme la plus aboutie de plusieurs types d'occupation possibles (habitat, occupation agraire ou pastorale, etc.).

Pour la seule période médiévale, les résultats de cette étude permettent de discerner trois phases essentielles dans la structuration territoriale de l'espace étudié (fig. 4) : une phase de développement et de mobilité du peuplement et des espaces agraires associés au cours des $\mathrm{VIII}{ }^{\mathrm{e}}-\mathrm{X}^{\mathrm{e}}$ siècles, une phase de recentrage au long des $\mathrm{XI}^{\mathrm{e}}$ $\mathrm{XV}^{\mathrm{e}}$ siècles, et enfin un nouvel essor lisible à partir de la seconde moitié du $\mathrm{XV}^{\mathrm{e}}$ siècle.

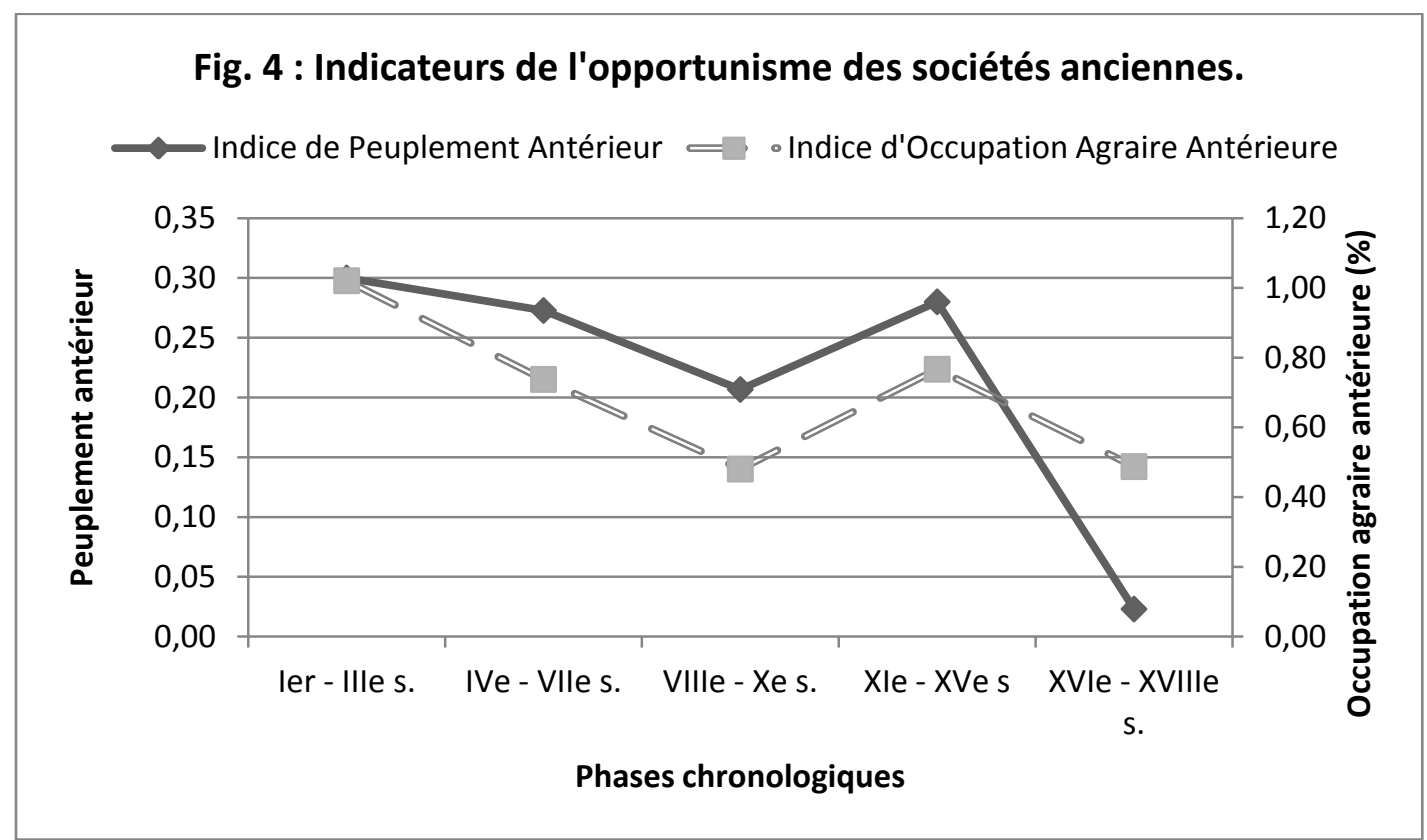

fig. 4 : Indicateurs de l’opportunisme des sociétés anciennes.

\section{L'essor du haut Moyen Âge (VIII ${ }^{e}-X^{e}$ siècles)}

\subsection{Le silence des textes}

On ne dispose malheureusement d'aucun texte avant la fin du $\mathrm{XI}^{\mathrm{e}}$ siècle. On pourra s'étonner de l'absence de mentions antérieures, émanant par exemple de cartulaires d'abbaye ou de diplômes carolingiens. Cette spécificité du Berry est handicapante pour qui s'intéresse au haut Moyen Âge et s'explique de plusieurs manières. Tout d'abord, et dès le XVII et le XVIII ${ }^{\mathrm{e}}$ siècle, les archives de l'abbaye de Déols, l'une des premières et des plus anciennes filiales de Cluny, et celles des abbayes 
de la Brenne (Méobecq et Saint-Cyran) ont disparu ${ }^{16}$. D'autre part, en 1859, un incendie a consumé une quinzaine de cartulaires entreposés dans le bureau du directeur des Archives départementales du Cher.

La fondation du chapitre Saint-Cyr de Sancergues est attribuée par la tradition historiographique à l'archevêque de Bourges saint Août (ou Aygulphe), au VIII' ${ }^{\mathrm{e}}$ siècle. Il s'agit d'une tradition locale qui n'est appuyée par aucun document d'archives. Selon sa vita, Aygulphe était un ermite vivant retiré au milieu des bois, dans la région de Sassierges (dans l'Indre), lorsqu'il fut élu évêque. Cependant, cette vita n'est qu'une légende qui ne mérite aucun crédit. Aygulphe est mort l'année même de son accession à l'épiscopat et son voisin, l'archevêque d'Orléans Théodulf, a laissé de lui un éloge en vers $^{17}$. On pourrait presque penser que la tradition qui attribue la fondation de la collégiale à saint Aygulphe ne repose que sur la proximité orthographique des deux noms de lieux de Sancergues et Sassierges, qui n'ont pourtant rien en commun...

L'existence du chapitre n'est réellement attestée qu'à partir de 1077. Le volume des Papsturkunden in Frankreich consacré au Berry ${ }^{18}$ ne recense que trois documents : un du pape Innnocent II (1140), un du pape Lucius III (1183) et un du pape Urbain III (1186). Ces trois documents sont conservés aux Archives départementales du Cher sous la même référence ${ }^{19}$. On trouve dans la même liasse un quatrième document, émanant du pape Grégoire VII et daté de 1077, qui n'est pas mentionné dans les Papsturkunden. Ces documents confirment des possessions du chapitre canonial, énumèrent des listes d'églises dépendant du chapitre, sans documenter les questions de tissu de peuplement de cette période dans la région de Sancergues.

\subsection{Développement et/ou mobilité du tissu de peuplement}

Les prospections menées entre 2003 et 2006 dans cette région ont permis d'identifier un premier cycle de peuplement dont le démarrage est lisible au cours de la protohistoire, connaît une forme d'apogée aux I ${ }^{\mathrm{er}}$-III ${ }^{\mathrm{e}}$ siècles et une phase de déprise aux $\mathrm{IV}^{\mathrm{e}}-\mathrm{VII}^{\mathrm{e}}$ siècles. La dynamique des espaces agraires associés aux établissements de

16. Devailly 1973, p. 11.

17. DeVAILly 1973, p. 85.

18. WIEDERHOLD 1910.

19. AD Cher, 11 G 10. 
chacune de ces phases chronologiques renforce cette image par la mise en évidence d'épisodes concordants d'extension et de rétraction de l'espace cultivé ${ }^{20}$.

À l'issue de ce premier cycle de peuplement qui semble prendre fin entre le $I^{\mathrm{e}}$ et le $\mathrm{VII}^{\mathrm{e}}$ siècle, un nouveau cycle démarre entre le VIII ${ }^{\mathrm{e}}$ et le $\mathrm{X}^{\mathrm{e}}$ siècle. L'image du peuplement pour la fin du haut Moyen $\hat{A} g e\left(\mathrm{VIII}^{\mathrm{e}}-\mathrm{X}^{\mathrm{e}}\right.$ siècles$)$ constitue la vraie surprise de nos campagnes de prospection. Il faut rappeler que les vestiges de cette période sont en général difficiles à détecter, la céramique du haut Moyen Âge étant dans cette région plutôt de mauvaise qualité et se détériorant rapidement dans le sol. Le haut Moyen Âge est donc souvent une phase apparaissant comme lacunaire dans les données de prospection au sol. Il n'en va pas ainsi pour la région de Sancergues. La phase VIII $-\mathrm{X}^{\mathrm{e}}$ siècles apparaît en effet comme la plus dynamique en termes d'occupation humaine, avec une densité d'établissements plus importante même que la période gallo-romaine qui est habituellement celle qui livre le plus de sites. En effet, dix établissements peuvent être datés de la période $\mathrm{VIII}^{\mathrm{e}}-\mathrm{X}^{\mathrm{e}}$ siècle, avec des choix d'implantation particuliers (fig.5). 


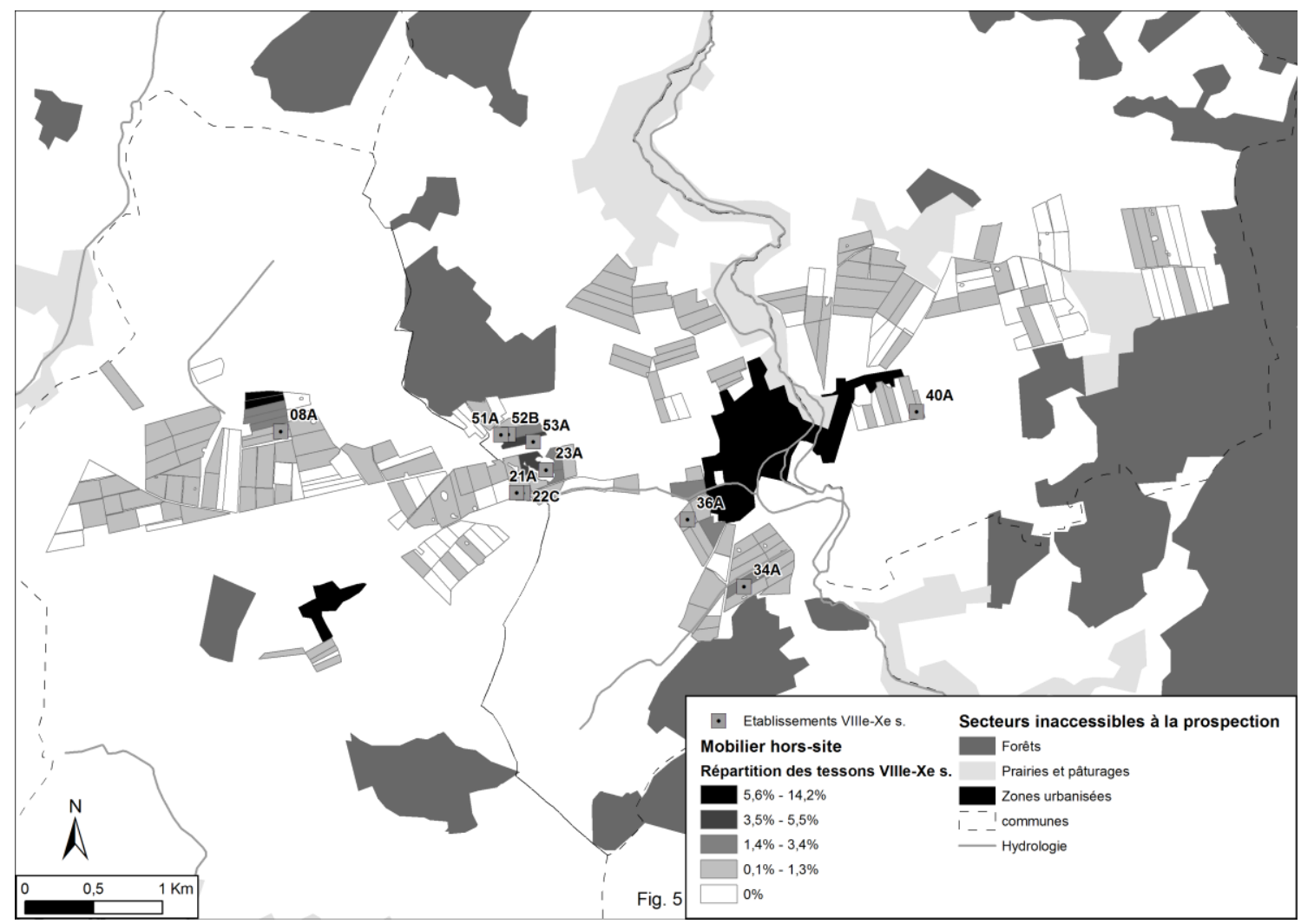

fig. 5 : Occupation du sol au VIIIe-Xe siècle d'après les sources archéologiques

Deux éléments d'importance sont à noter: d'abord, l'occupation intensive $d u$ secteur central des buttes d'argiles à silex, avec six établissements implantés dans ce seul secteur, dont l'un présente une superficie très importante de l'ordre de 1,7 ha $\left(n^{\circ} 23 \mathrm{~A}\right)$; ensuite, l'implantation d'un site de cette période sur la commune de SaintMartin-des-Champs ( $\left.{ }^{\circ} 40 \mathrm{~A}\right)$. Cet établissement est le plus ancien repéré sur cette commune. Il semble donc que l'habitat progresse sensiblement vers l'est de notre secteur (fig. 5).

Ce constat de la forte occupation du haut Moyen Âge doit être souligné. Cette densité s'appuie également sur des sites assez aisés à repérer, notamment par des concentrations très importantes de mobilier. Par exemple, l'un des établissements $\left(\mathrm{n}^{\circ}\right.$ 08A) a livré un lot de plus de 900 tessons, ce qui est tout à fait exceptionnel pour la période.

Les deux indices d'opportunisme (indice de peuplement antérieur et indice de mise en valeur agraire antérieure) sont particulièrement bas au cours de cette phase. On constate d'une part de nombreuses implantations sur de nouveaux sites : seuls deux établissements sur dix sont des réoccupations de sites habités antérieurement; d'autre 
part, ces nouvelles implantations interviennent souvent au sein de nouveaux espaces, très peu mis en valeur avant cette date. Cette phase apparaît donc comme celle où les implantations sont les moins opportunistes - ou les plus novatrices - dans leurs choix spatiaux.

\subsection{Mise en valeur de nouveaux terroirs}

Le haut Moyen Âge (VIII ${ }^{\mathrm{e}}-\mathrm{X}^{\mathrm{e}}$ siècle) apparaît également comme un pic dans la courbe d'évolution de la densité moyenne de mobilier hors-site récolté (fig. 6). Cette valeur atteint près de 4 tessons par hectare, soit la plus grande densité de mobilier observée jusque-là dans cette région. Cette valeur n'est dépassée que pour les périodes modernes et contemporaines. La densité de mobilier observée pour la phase antérieure est multipliée par plus de 10 . On peut interpréter cette augmentation comme le signe d'un recours plus régulier à l'épandage de fumures dans les parcelles cultivées.

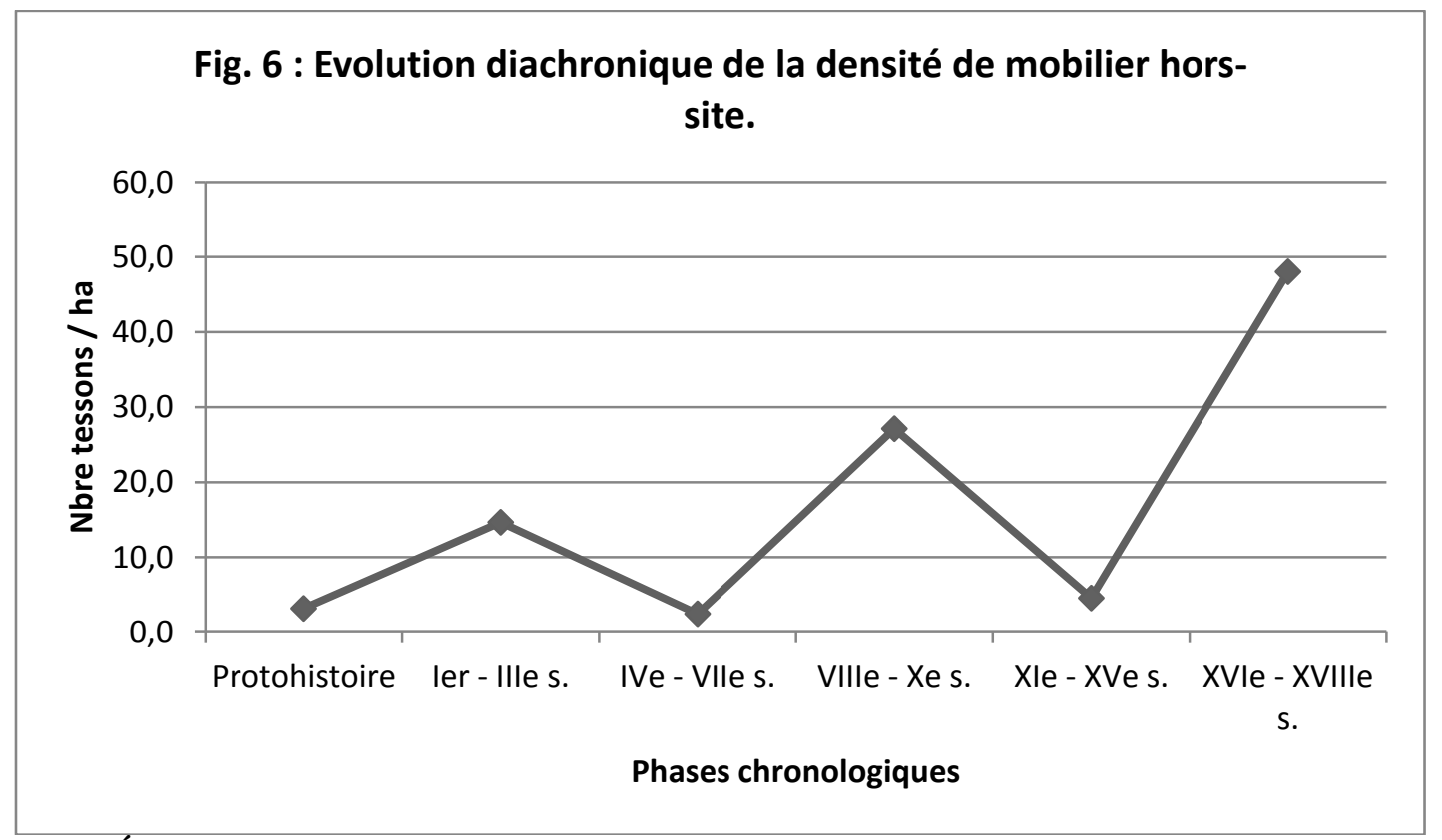

fig. 6 : Évolution diachronique de la densité de mobilier hors-site.

Cet essor agricole est également lisible dans l'augmentation de la surface ayant reçu des amendements agraires. Dans l'observation diachronique de l'évolution de la superficie amendée, on distingue la superficie totale amendée et la superficie 
intensément amendée, c'est-à-dire présentant des densités de mobilier supérieures à la moyenne de la période (fig. 7).

La confrontation de ces deux indicateurs sur un même graphe fournit une troisième information. Les variations de l'écart entre les deux courbes peuvent être lues comme une illustration de la variation de concentration ou de dispersion des espaces amendés. Plus les deux courbes sont proches, plus les champs anciennement amendés sont concentrés sur un espace restreint ; à l'inverse, plus les courbes s'écartent l'une de l'autre, plus les amendements sont dispersés dans l'espace prospecté ${ }^{21}$.

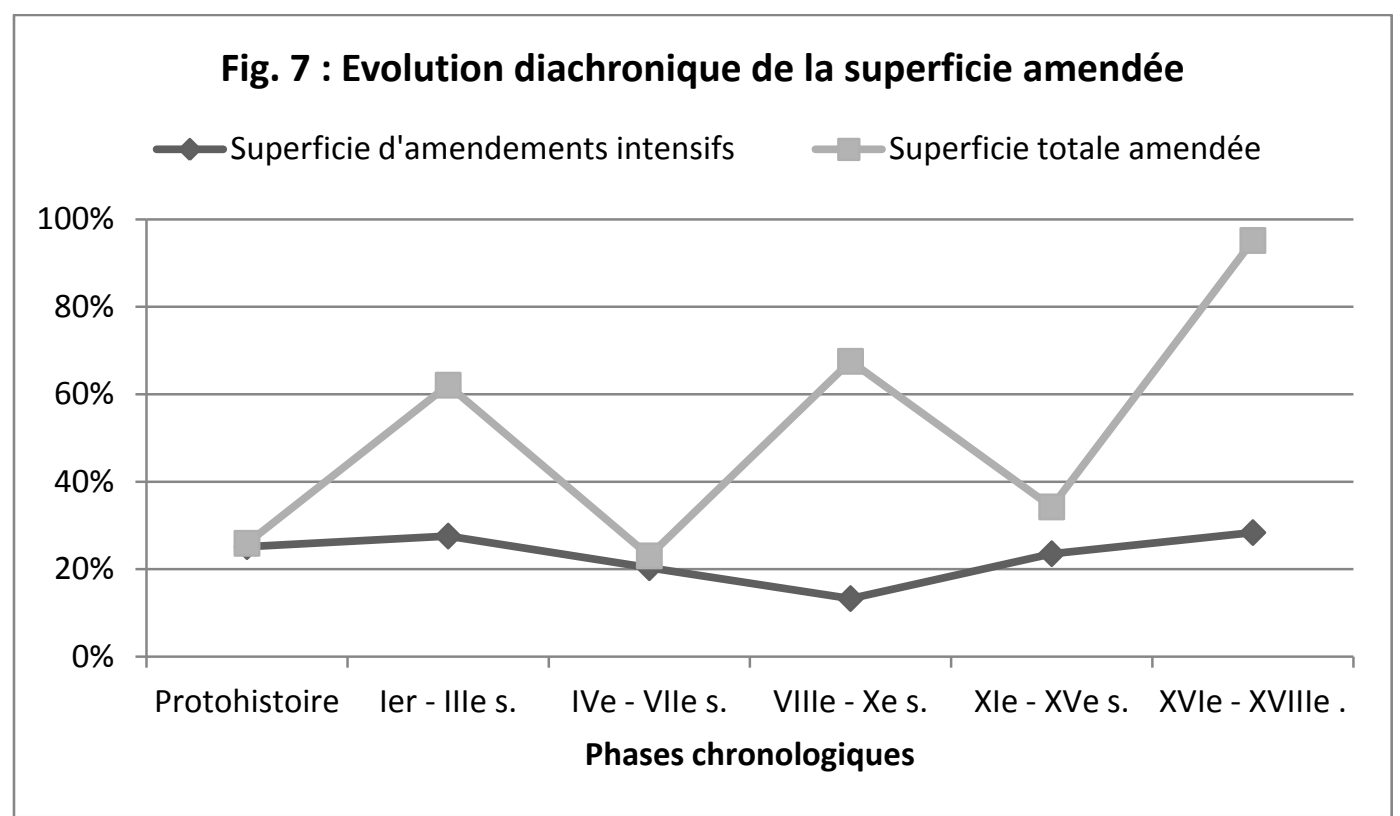

fig. 7 : Évolution diachronique de la superficie amendée.

Les VIII', IXe et $\mathrm{X}^{\mathrm{e}}$ siècles donnent l'image d'un important essor de la superficie totale occupée, qui dépasse le niveau atteint pour la période romaine ( ${ }^{\mathrm{er}}-\mathrm{III}^{\mathrm{e}}$ siècle), avec $67,5 \%$ des espaces prospectés ayant livré des traces d'amendements agraires. En revanche, la part des espaces intensivement amendés atteint son niveau le plus bas à $13,3 \%$ des espaces prospectés, au terme d'une diminution amorcée à la période romaine. Il apparaît donc que l'espace agraire des $\mathrm{VIII}^{\mathrm{e}}-\mathrm{X}^{\mathrm{e}}$ siècles est très homogène, sans traitements différenciés très marqués dans les modes d'amendement.

La période connaît également des préférences dans la localisation des espaces exploités. En particulier, on note la recherche des versants exposés au nord. Ces espaces

21. Cette interprétation en termes de dispersion/concentration des espaces cultivés a été validée par la mise en œuvre d'indicateurs statistiques spatiaux appropriés (POIRIER 2006c). 
représentent alors $14 \%$ des espaces amendés alors qu'ils ne concernent que $5 \%$ des surfaces prospectées. Les versants exposés à l'ouest et au sud-est sont sous-représentés. Les terrains pentus sont également peu investis : la proportion de terrains présentant un pendage supérieur à $2 \%$ est alors de $30 \%$. Les sols de texture légère (type 10 de la classification adoptée par le PCR Berry ${ }^{22}$ ) sont amendés de manière importante, tandis que les sols lourds et acides (type 9 de cette même classification) semblent délaissés. Ils ne constituent que $3 \%$ des espaces exploités. On remarque une nette préférence pour les espaces proches d'un cours d'eau (à moins de $400 \mathrm{~m})^{23}$.

\section{Une polarisation au Moyen Âge central ?}

\subsection{Des occupations opportunistes.}

Le Moyen Âge central et final $\left(\mathrm{XI}^{\mathrm{e}}-\mathrm{XV}^{\mathrm{e}}\right.$ siècle) est assez difficile à identifier archéologiquement, car il n'est représenté dans ce secteur que par un seul type de céramique, dont la datation semble d'ailleurs devoir être limitée au XI ${ }^{\mathrm{e}}-\mathrm{XIII}{ }^{\mathrm{e}}$ siècle pour l'essentiel du mobilier recueilli. Il y a clairement un problème de hiatus chronologique dans la mesure où très peu de vestiges de la fin du Moyen Âge $\left(X V^{\mathrm{e}}-\mathrm{XV}^{\mathrm{e}}\right.$ siècle) ont été récoltés. Cette période $\left(\mathrm{XIV}^{\mathrm{e}}-\mathrm{XV}^{\mathrm{e}}\right.$ siècle) se manifeste dans la région ${ }^{24}$ par des tessons glaçurés dont nous n'avons trouvé que très peu d'exemples dans la zone étudiée. Toutefois, la glaçure ayant pu disparaître à cause de l'altération des tessons due aux pratiques agraires, la datation de ce type de céramique a été élargie aux $X{ }^{\mathrm{e}}-X V^{\mathrm{e}}$ siècles.

Les sites identifiés en prospection présentant une occupation $\mathrm{XI}^{\mathrm{e}}-\mathrm{XV}^{\mathrm{e}}$ siècle sont au nombre de quatre (fig. 8). Il s'agit d'établissements ayant fonctionné à cette période et abandonnés depuis. L'image archéologique du peuplement pour cette phase est dominée par deux établissements principaux. L'important établissement qui avait livré plus de 900 tessons pour la phase $\mathrm{VIII}^{\mathrm{e}}-\mathrm{X}^{\mathrm{e}}$ siècles repéré sur la commune de Charentonnay est encore occupé au cours du Moyen Âge central (n08B). L'autre structure de plus d'1,5 ha est également occupée au cours de cette période ( $\left.\mathrm{n}^{\circ} 23 \mathrm{~B}\right)$; elle

22. BATARDY, BUChSENSCHÜTZ et DUMASY 2001, p. 47.

23. POIRIER 2007, fig. 74-77, p. 349-350.

24. HUSI 2003. 
est d'ailleurs la seule qui subsiste du petit groupement d'habitats observés à la période précédente dans ce secteur des bas de pentes d'argiles à silex.

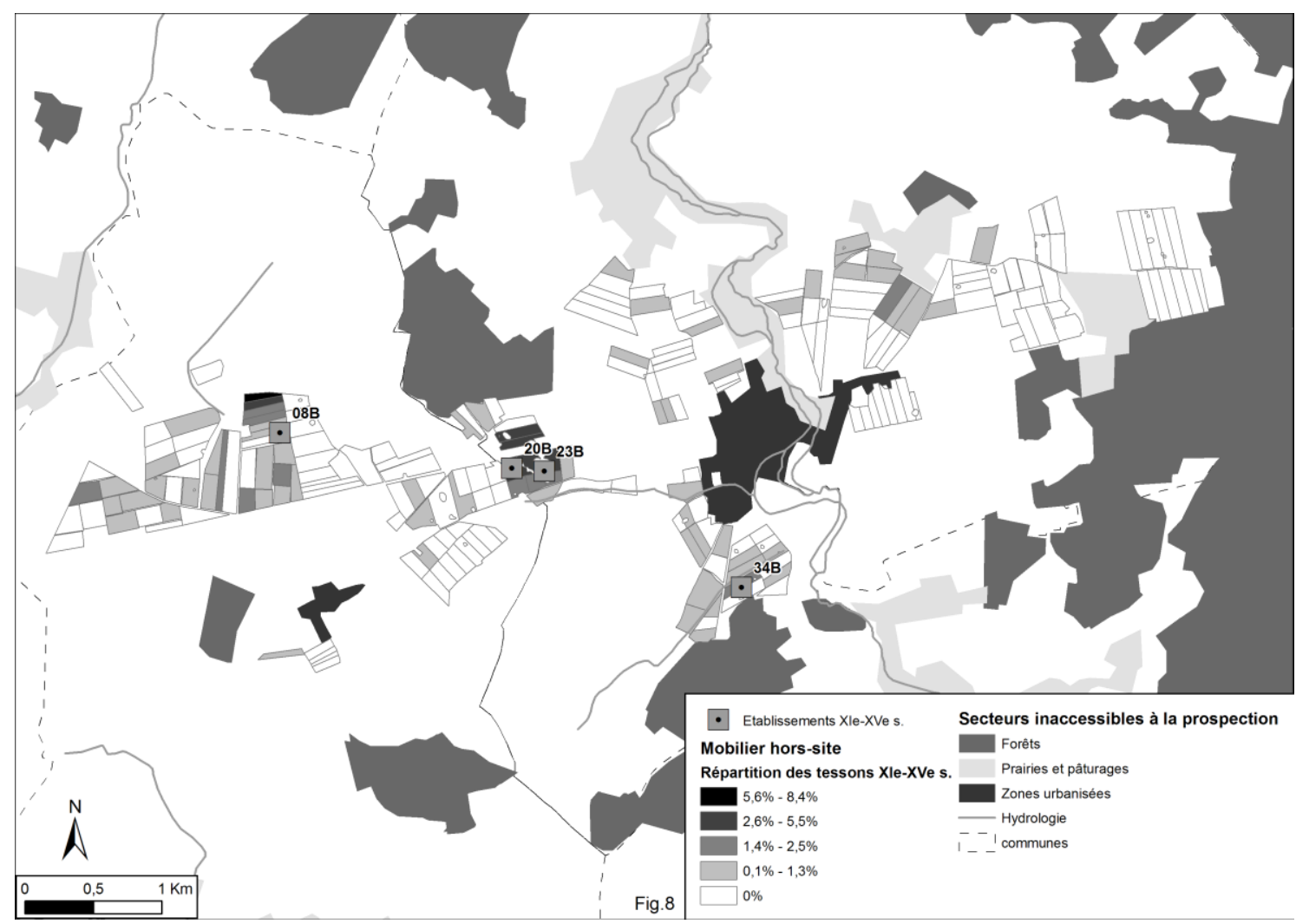

fig. 8 : Occupation du sol au XIe-XVe siècle d'après les sources archéologiques.

L'opportunisme augmente au cours de cette période. On retrouve alors les niveaux atteints au cours de la période romaine et surtout de l'Antiquité tardive (fig. 4). Il faut souligner qu'aucune implantation nouvelle n'est réalisée au cours de cette phase et que les établissements occupés sont au cœur de terroirs déjà mis en valeur de longue date. Le secteur central des buttes résiduelles d'argiles à silex est celui qui concentre le plus d'habitats. Les établissements de ce secteur comptent dix implantations antérieures dans un rayon de $500 \mathrm{~m}$. Il y a donc peu d'initiative nouvelle en matière de peuplement au cours de cette phase.

\subsection{Une image de stabilité livrée par les sources écrites}

À partir de cette période, la seule représentation des sites archéologiques n'est pas suffisante. En effet, à partir du $\mathrm{XI}^{\mathrm{e}}$ siècle, on dispose dans ce secteur d'archives mentionnant un certain nombre de lieux habités, dont certains le sont encore. Un grand 
nombre de hameaux actuels apparaissent dans les textes et doivent être pris en compte, avec les données archéologiques, dans l'étude de l'occupation du sol. L'agglomération de Sancergues par exemple, le hameau de Deux-Lions à Saint-Martin-des-Champs, ou celui de Villeret à Charentonnay apparaissent dans les textes entre le $\mathrm{XI}^{\mathrm{e}}$ et le $\mathrm{XIII}^{\mathrm{e}}$ siècle (fig.9).

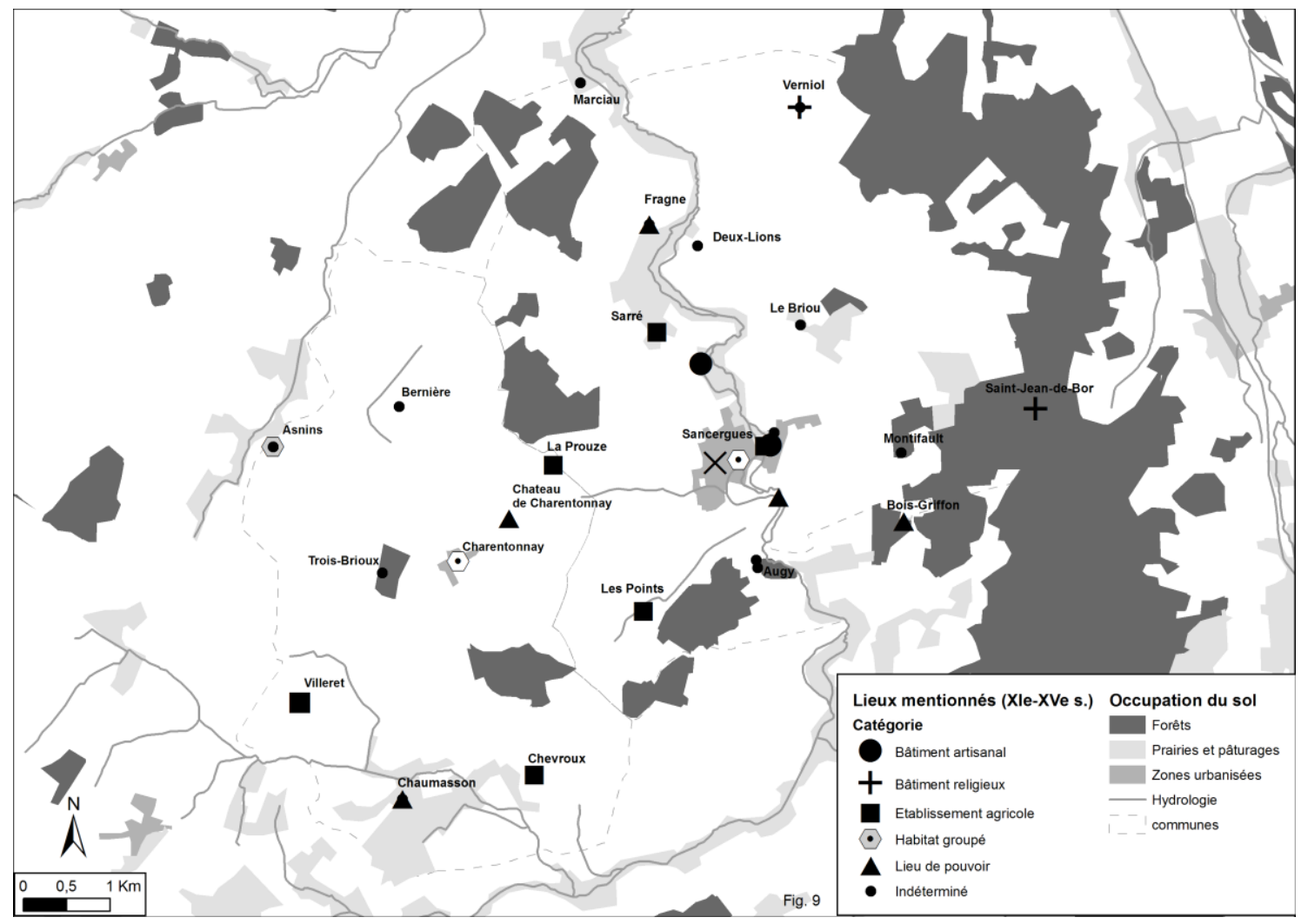

fig. 9 : Lieux mentionnés dans les sources écrites du XIe au XVe siècle.

Le dépouillement des sources écrites de cette période conduit à poser l'hypothèse d'une apparente fixation précoce et durable d'une partie des principaux points de peuplement actuels. En effet, à une exception près, les localités mentionnées dans les actes du $\mathrm{XII}^{\mathrm{e}}$ au $\mathrm{XV}^{\mathrm{e}}$ siècle sont encore des points de peuplement existant au début du XIX ${ }^{\mathrm{e}}$ siècle. Même si l'image du peuplement livrée par ces documents n'est pas exhaustive, elle semble assez proche de celle que l'on peut lire sur le cadastre napoléonien. Cette observation permettrait de conclure que la fixation des principaux points de peuplement subcontemporains a été relativement précoce, puisque effective au $\mathrm{XII}^{\mathrm{e}}$ ou $\mathrm{XIII}^{\mathrm{e}}$ siècle, et durable, dans la mesure où la documentation ne permet d'enregistrer aucun abandon. 


\subsection{Rétraction de l'espace agraire et intégration de contextes agricoles plus contraignants}

La pression agraire observée pour le $\mathrm{VIII}^{\mathrm{e}}-\mathrm{X}^{\mathrm{e}}$ siècle ne semble pas se maintenir au $\mathrm{XI}^{\mathrm{e}}-\mathrm{XV}^{\mathrm{e}}$ siècle qui montre un retour à de faibles densités de mobilier d'épandage, de l'ordre de 0,58 tessons par hectare.

Les $\mathrm{XI}^{\mathrm{e}}-\mathrm{XV}^{\mathrm{e}}$ siècles apparaissent comme une nouvelle phase de recentrage de l'espace agraire autour des secteurs cultivés de manière intensive. On observe en effet un recul de la superficie totale occupée ou fréquentée, mais une nette reprise des espaces intensivement exploités qui atteignent $23 \%$ des espaces prospectés. L'évolution observable pour cette période est très proche de celle de la transition entre Antiquité tardive et haut Moyen Âge $\left(\mathrm{IV}^{\mathrm{e}}-\mathrm{VII}^{\mathrm{e}}\right.$ siècles) qui se présente également comme une phase de recentrage succédant à une importante phase d'essor.

Les espaces intensément amendés au cours de cette période favorisent les versants exposés au sud-sud-ouest et dans une moindre mesure ceux exposés au nord-nord-ouest. Les versants exposés à l'ouest semblent toujours délaissés. Les pentes ne paraissent plus contraignantes dans la mesure où l'équirépartition est quasiment atteinte pour la classe des pentes comprises entre 2 et $4 \%$ de déclivité. Les sols exploités majoritairement sont toujours les plus favorables (types 10 et 11), mais on note une augmentation de la part des sols contraignants (type 9), qui restent malgré tout sous-représentés. Le rapport aux cours d'eau change au cours de cette période. L'immédiate proximité n'est plus systématiquement favorisée. Les classes de distances 700-800 m et 900-1000 m sont sur-représentées dans le profil des espaces amendées ${ }^{25}$.

\section{La reprise du Moyen Âge final et de la période moderne (fin $X V^{e}-X V I I I^{e}$ siècles)}

\subsection{Un nouvel essor du peuplement}

Six établissements datables des XVIe-XVIIIe siècles ont été identifiés en

25. POIRIER 2007, fig. $78-81$, p. 351-352. 
prospection (fig. 10). Leur position est particulière car ils sont implantés sur des espaces encore très peu occupés jusqu'à cette date. C'est le cas des structures identifiées sur la commune de Charentonnay qui sont les premières à s'installer sur les versants ouest des buttes d'argiles à silex, plutôt répulsifs jusqu'à cette date. C'est également le cas des occupations identifiées à l'extrême est de la commune de Saint-Martin-des-Champs qui ont livré des tessons de grès ainsi que des fragments de tuiles et de briques. Ils peuvent aussi bien correspondre à des bâtiments d'exploitation qu'à des fermes. Ils témoignent d'une progression vers l'est de l'occupation, sur des terrains peu favorables à l'agriculture, là aussi constitués d'argiles et de limons lourds à mettre en valeur. L'image du peuplement est incomplète car il faut y ajouter la plupart des hameaux actuels qui existent à coup sûr à cette période, comme les hameaux de la Justellerie et de la Perronerie, la ferme de la Bernière, le hameau de Sarré, le hameau de la Tuilerie, etc.

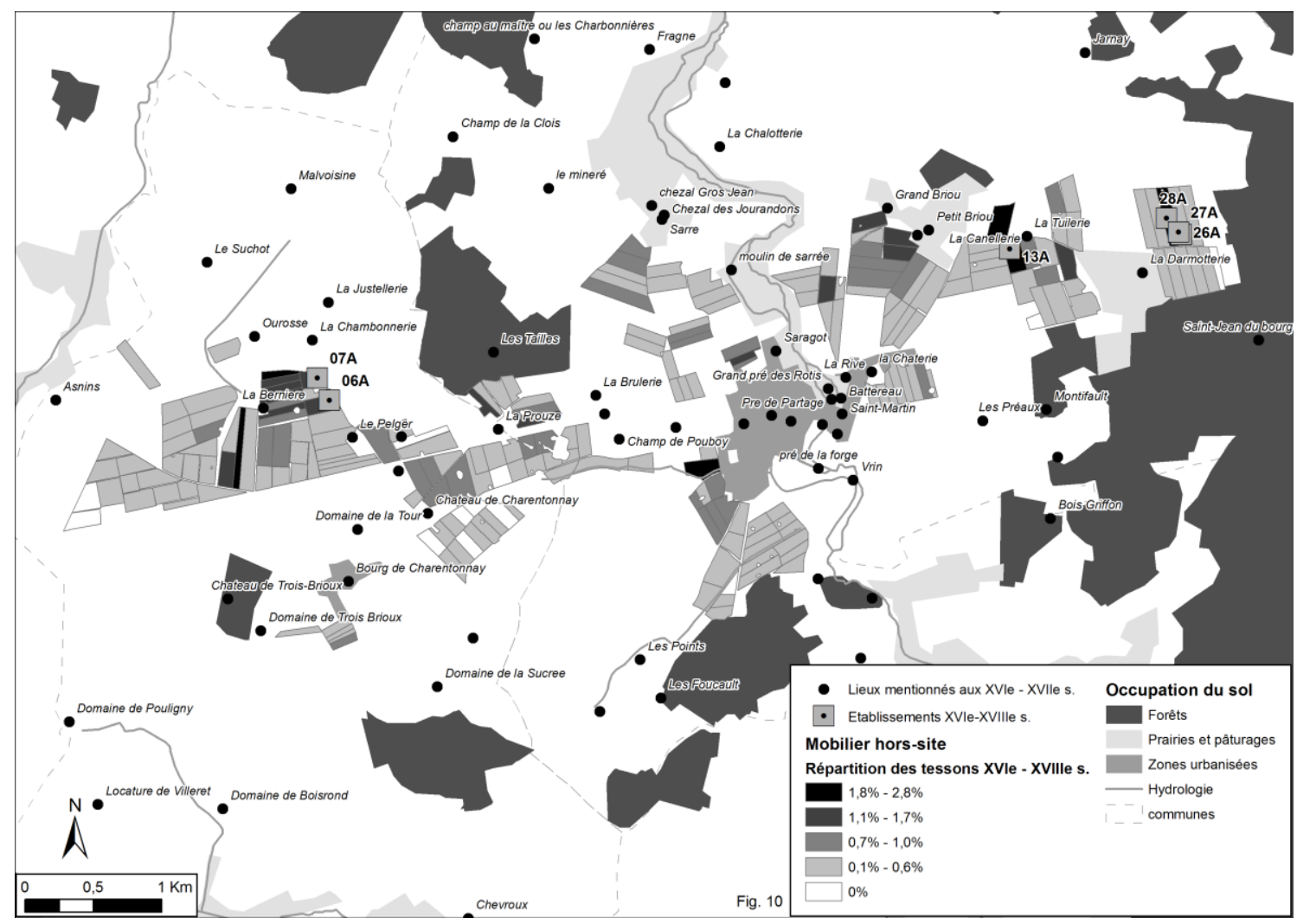

fig. 10 : Occupation du sol au XVIe-XVIIIe siècle d'après les sources écrites et archéologiques. 
Archéologie Médiévale, t.40 (2010), p.15-32

\subsection{Extension des espaces cultivés et intensification des amendements}

Cette phase montre une reprise très nette de l'amendement extensif, dans la mesure où la superficie totale des espaces ayant livré des traces d'occupation atteint $95 \%$ des espaces prospectés. Dans le même temps, les espaces exploités de manière intensive poursuivent leur reprise et représentent $28 \%$ des espaces prospectés.

On peut considérer le redémarrage du $\mathrm{XVI}^{\mathrm{e}}-\mathrm{XVIII}{ }^{\mathrm{e}}$ siècle comme une véritable explosion de l'intensité des amendements agraires. La densité moyenne de mobilier hors-site récolté atteint alors 5,56 tessons par hectare. Les quantités observées sont sans commune mesure avec les phases antérieures qui n'avaient pas dépassé, ou de manière exceptionnelle, les quatre tessons par hectare pour le VIII $-\mathrm{X}^{\mathrm{e}}$ siècle.

La période moderne se caractérise également par des choix d'implantation très peu marqués. Globalement, les quelques préférences lisibles concernant l'exposition des versants montrent une faveur pour les orientations au nord-nord-est et au sud-sud-ouest, mais aucun contexte n'est réellement proscrit. Les pentes les plus fortes sont néanmoins délaissées et une nette préférence est accordée aux terrains les moins pentus (65\% des espaces exploités présentent une pente inférieure à $2 \%$ ). Pour la première fois, les sols les plus favorables ne sont pas sur-représentés. On observe une répartition des espaces amendés sur les différents types de sols proportionnellement à leur représentation dans les zones prospectées. L'évolution du rapport aux cours d'eau amorcée au cours de la phase précédente se poursuit au $\mathrm{XVI}^{\mathrm{e}}-\mathrm{XVIII}^{\mathrm{e}}$ siècle dans la mesure où l'immédiate proximité est désormais rejetée (les classes inférieures à 200 m sont sous-représentées). Les espaces amendés se concentrent à des distances comprises entre 200 et $500 \mathrm{~m}$ pour l'essentiel $^{26}$.

\subsection{Le rôle des acteurs institutionnels dans la « reconstruction »}

Les sources écrites invitent en outre à voir dans cette période un épisode de grande mobilité du peuplement. La documentation est certes beaucoup plus nombreuse

26. POIRIER 2007, fig. $82-85$, p. 353-354. 
et variée (avec les premiers terriers ${ }^{27}$ ), mais l'on croit pouvoir y lire des évolutions propres à cette période. À partir du $\mathrm{XVI}^{\mathrm{e}}$ siècle, des points de peuplement sont mentionnés, pour l'essentiel des établissements agricoles isolés, qui n'ont pas perduré au-delà du XVII ${ }^{\mathrm{e}}$ siècle pour plusieurs d'entre eux. Certains n'ont pas pu être localisés dans la mesure où le toponyme même avait disparu du corpus microtoponymique du cadastre napoléonien. On dénombre au total 19 localités mentionnées au XVI ${ }^{\mathrm{e}}$ siècle et absentes du cadastre napoléonien. Toutefois, une petite part des points de peuplement qui apparaissent à la fin du Moyen Âge ne sont pas des établissements agricoles voués à l'échec. On peut en particulier restituer les étapes du développement de certains hameaux grâce à une documentation particulièrement riche, émanant du chapitre canonial. On y entrevoit d'ailleurs le rôle déterminant des chanoines à l'initiative desquels certains espaces sont mis en valeur à cette période.

Observons tout d'abord le processus d'apparition du hameau des Points, sur l'actuelle commune de Sancergues. Le premier acte date de 1467, lorsque les chanoines de Sancergues arrentent deux mouhées de terre (une dizaine d'hectares) au lieu-dit « la Fontaine Morte » mentionné comme étant situé «dans les bois de Tannois », à deux frères nommés Poings ${ }^{28}$. Il faut d'ailleurs souligner que l'utilisation de la «mouhée » pour évaluer la surface arrentée est caractéristique en Berry d'un espace à valoriser, comme l'a remarqué F. Michaud-Fréjaville :

" L'utilisation des mouées, tout comme celle d'un nombre élevé de sestérées ou d'arpents, est donc toujours à examiner de très près : dans bien des cas on se rend compte que ces mesures ont été employées pour un ensemble à aménager et non pas en exploitation et que nous les rencontrons au moment de l'attribution à un nouveau tenancier d'un bloc en «bois et buissons » ou en «pâturages et hermes », ou encore en «terres et bruyères ${ }^{29} » "$.

En échange de cette terre, les preneurs ont l'obligation de construire au moins une maison ou une grange.

En 1493, un nouveau bail perpétuel est conclu entre les chanoines de Sancergues et les frères Poings. Le bail porte cette fois sur une surface de deux mouhées et demie. L'acte précise que dans cet « héritage et tènement lesdits Poings frères ont basti et édifié chacun une maison et pour iceux une grange ${ }^{30} »$. En un peu moins de vingt-cinq ans, les

27. AD Cher, E15; $11 \mathrm{G} 105$.

28. AD Cher, $11 \mathrm{G} 16$.

29. MichaUd-FREJAVILLE 1997, p. 127.

30. AD Cher, $11 \mathrm{G} 16$. 
preneurs ont donc mis en valeur ces deux mouhées et demie de terre en les défrichant et en y construisant deux maisons et une grange. Cet acte de 1493 permet de s'assurer de la localisation des biens mentionnés puisque des confronts très précis sont cités.

On peut suivre de la même manière l'émergence d'un habitat situé à proximité du hameau des Points, à $250 \mathrm{~m}$ au sud-est : le lieu des Foucaults. En 1556, les chanoines de Sancergues vendent à quatre preneurs de la famille Foucault « une pièce de terre moitié en labour moitié bois et buissons située au dessus de l'héritage des Points ${ }^{31}{ }$. Il semble que cet espace ait été rapidement mis en valeur, puisqu'en 1575 «Pierre Fossard [...] délaisse à Noël Foucault [...] et à Jean Colinet [...] la quatrième partie du chezal des Foucaults, consistant en maison, grange, bergerie, ouche, terres labourables, bois et buissons $^{32}{ }$. Des bâtiments ont donc été implantés, puisqu'aucun n'était mentionné dans l'acte de 1556. Les confronts cités dans chacun des actes permettent de s'assurer que l'on a affaire au même tènement. La famille Foucault poursuit sa politique d'acquisition dans ce secteur par différents actes passés entre 1578 et $1598^{33}$. Il faut d'ailleurs noter que les deux points de peuplement ainsi créés (les Points et les Foucaults) trouvent l'origine de leur nom dans le patronyme de ceux qui ont défriché ces terres.

\section{Du micro au macro : une mise en perspective}

En guise de conclusion, il paraît utile de replacer la microrégion étudiée dans le contexte géographique plus large du Berry médiéval. On ne dispose guère que de deux synthèses régionales pour décrire les modèles d'évolution élaborés à macro-échelle, et essentiellement à partir des sources écrites. Il s'agit de l'ouvrage de G. Devailly, Le Berry du X $X^{e}$ au milieu du XIII siècle paru en 1973, et la thèse d'État de F. MichaudFréjaville, Économie et vie rurales en Berry à la fin du Moyen Âge soutenue à l'Université de Paris I en 1997. Il faut souligner d'emblée l'inégale valeur de ces deux études, que 25 ans séparent au cours desquels l'essentiel des paradigmes qui soutenaient l'histoire du peuplement médiéval - et particulièrement du haut Moyen Âge - ont été remis en cause à la suite du développement de l'archéologie préventive. Les deux 
approches poursuivent d'ailleurs des objectifs différents : faire l'histoire institutionnelle de la féodalité pour G. Devailly et celle de l'économie rurale pour F. MichaudFréjaville. Les évolutions intervenant dans l'occupation du sol ne sont donc pas l'objet d'étude principal de ces deux ouvrages.

Pour autant, il est nécessaire de tenter une évaluation de la représentativité des observations réalisées à microéchelle dans la région de Sancergues. Il est également précieux de prendre la mesure des apports de l'archéologie extensive documentée par la prospection au sol.

\subsection{Remise en cause d'un modèle misérabiliste établi pour le haut Moyen Âge}

On peut interpréter la fin du haut Moyen Âge comme une période de relative prospérité lisible dans la dynamique du paysage et du peuplement dans la région de Sancergues. On a vu que cette période $\left(\mathrm{VIII}^{\mathrm{e}}-\mathrm{X}^{\mathrm{e}}\right.$ siècle) correspondait à un épisode d'optimum par le nombre d'établissements occupés comme par l'extension des espaces cultivés. Cette observation va à l'encontre du schéma proposé à partir des seules sources écrites par G. Devailly qui ne décèle «aucune révolution agricole, aucun progrès technique important entre le $\mathrm{IX}^{\mathrm{e}} \mathrm{s}$. et le troisième quart du $\mathrm{XI}^{\mathrm{e}} \mathrm{s}$. On continue à cultiver les mêmes terres que dans les siècles passés et on ne cultive guère qu'elles. Aucun indice ne permet de déceler d'importants défrichements durant ces deux siècles ${ }^{34}{ }^{3}$. Cette image est caractéristique du tableau généralement misérabiliste dressé pour le haut Moyen Âge jusque dans les années 1990, avant la prise de conscience, suscitée par l'archéologie préventive, du dynamisme du peuplement lisible à cette période ${ }^{35}$. Cette image est une construction historiographique qui s'appuie sur un hiatus des sources écrites particulièrement aggravé en Berry. Le département du Cher a d'ailleurs fait l'objet d'une expérience pionnière d'investigation archéologique systématique sur le tracé de l'autoroute $\mathrm{A} 71^{36}$ qui a permis, dès le milieu des années 1980, de démontrer la réalité du peuplement du haut Moyen Âge et ainsi de remettre en cause cette image de « désert ».

34. DeVAiLly 1973, p. 221.

35. LORREN et PERIN 1995.

36. FERDIERE et RIALLAND 1994 ; FERDIERE et RIALLAND 1995 ; FERDIERE et RIALLAND 1996. 
Cette période apparaît dans la région de Sancergues, à la lumière des données archéologiques, comme une des deux plus dynamiques en matière d'initiative agraire, avec la période moderne. Nos prospections démontrent donc le dynamisme du milieu rural à cette période.

\subsection{Essor ou restructuration au plein Moyen Âge ?}

G. Devailly voit dans la période $\mathrm{du} \mathrm{XI}^{\mathrm{e}}$ au $\mathrm{XIV}^{\mathrm{e}}$ siècle une phase d'extension importante des terres arables, fondée sur l'examen des terroirs et de la toponymie qui semblent indiquer des épisodes de défrichements. Son argumentation n'est toutefois pas développée et les insuffisances des sources sont largement soulignées. Il conclut pourtant à un grand mouvement d'extension des terres labourables en Berry du $\mathrm{XI}^{\mathrm{e}}$ au $\mathrm{XIV}^{\mathrm{e}}$ siècle sans pouvoir en préciser ni les modalités, ni les phases.

La seule image archéologique du paysage agraire de cette période dans la région de Sancergues ne permet pas de dresser le même bilan. Cette phase (XI ${ }^{\mathrm{e}}-\mathrm{XV}^{\mathrm{e}}$ siècles) y est marquée par une baisse conjointe de la superficie amendée et de l'intensité de ses amendements. C'est également la phase au cours de laquelle on observe le moins de mise en valeur d'espaces nouveaux. Cette période a été interprétée plus haut comme un épisode de consolidation des terroirs nouvellement mis en valeur au cours de la phase précédente. Évidemment, cette image archéologique est partielle, puisqu'elle ne donne à voir que l'étendue des espaces ayant bénéficié d'amendements agraires. D'autres types de mise en valeur peuvent exister et ne laisser aucune trace archéologique. On peut toutefois considérer que si cette période avait réellement connu une extension notable des terres arables à Sancergues, cette évolution se serait traduite d'une manière ou d'une autre dans la répartition des épandages agraires.

La fin de cette période en revanche (mi XIV ${ }^{\mathrm{e}}-\mathrm{mi} \mathrm{XV}^{\mathrm{e}}$ siècle) est décrite comme un épisode de marasme économique lié aux troubles engendrés par la guerre de Cent Ans ${ }^{37}$. La vallée de la Loire a d'ailleurs été particulièrement concernée pendant un quart de siècle, avec notamment la prise de la ville de Sancerre en $1361^{38}$. On a d'ailleurs un témoignage anecdotique de cette période troublée par la découverte d'un trésor

37. MichaUd-FREJAVILLE 1997, p. 432.

38. Ibid, p. 432. 
monétaire près du hameau des Buriaux (commune de Sancergues) ${ }^{39}$.

La région de Sancergues, située en plein cœur du Val de Loire, a nécessairement eu à subir ces passages de troupes et ces pillages mais, sans en minimiser l'importance, on ne peut certainement pas leur attribuer l'origine entière des évolutions observées dans la dynamique du peuplement et des espaces agraires. Ces épisodes de violence ne peuvent sans doute pas à eux seuls expliquer la réorganisation des terroirs et de l'habitat. Si la Grande Peste peut être également une explication séduisante, il faut souligner ici les difficultés méthodologiques inhérentes à l'exercice de confrontation des observations archéologiques faites à micro-échelle avec les tendances de fond qui affectent la démographie. Les évènements militaires, migratoires ou sanitaires sont documentés à une échelle de temps bien plus fine (quelques années ou décennies) que les données archéologiques de prospection (précision de quelques siècles). La seule observation de la baisse du nombre de sites archéologiques ou de la rétraction des espaces agraires n'est pas suffisante pour conclure à une importante baisse démographique directement liée à ces évènements, dans la mesure où l'on ne sait rien de la taille et de la population des sites subsistants, et que rien dans les sources écrites locales ne nous informe sur ce point. Il semble plutôt que le tissu de peuplement et les espaces agraires associés arrivent à ce moment à la fin d'un « cycle » de croissance que les événements politiques, militaires et sanitaires n'ont fait qu'aggraver. Après l'épisode d'importante initiative agraire et de peuplement des VIII ${ }^{\mathrm{e}}-\mathrm{X}^{\mathrm{e}}$ siècles, le Moyen Âge central est en effet marqué par une stabilisation des noyaux de peuplement autour de ceux qui, pour la plupart, sont encore occupés aujourd'hui. L'emprise des espaces amendés a diminué et s'est recentrée autour de quelques pôles. Cela n'exclut toutefois pas d'autres formes d'occupation du sol, comme un développement possible des prés, des pâturages, ou de terres arables non fumées.

\subsection{Les signes d'une reconstruction au Moyen Âge final}

Après 1450 , le Berry connaît un important mouvement de mise en culture de certains terroirs, avec des bonifications de terres, des défrichements, des regroupements de cultures (vignes et prés), des creusements d'étangs, des implantations de nouveaux

39. «Bulletin Numismatique », Mémoire de la Société des Antiquaires du Centre, t. 12, 1882-1883, p. 294-295 
hameaux avec l'édification de maisons. On constate alors à l'échelle régionale un rôle croissant des grandes exploitations et la place du mouton et de l'élevage en leur sein, l'augmentation de la place de la vigne et des chènevières dans les terroirs villageois, ainsi que l'attaque des zones périphériques des finages paroissiaux et seigneuriaux ${ }^{40}$. Ce mouvement est interprété comme une restauration des terres correspondant aux récupérations démographiques.

La région de Sancergues s'inscrit tout à fait dans ce schéma par le constat d'un important épisode de mise en valeur agraire au cours de la phase $\mathrm{XVI}^{\mathrm{e}}$-XVIII ${ }^{\mathrm{e}}$ siècle, qui se manifeste d'après les données de prospection par une augmentation impressionnante de la surface amendée et une progression forte de l'intensité de ces amendements. Le mouvement de mise en valeur de nouveaux espaces est également bien lisible par un déplacement des espaces cultivés sur des terrains jusque-là peu intensément exploités. Cette expansion agricole est accompagnée d'une nouvelle vague de peuplement que les sources écrites permettent de faire débuter dans la seconde moitié $\mathrm{du} \mathrm{XV}^{\mathrm{e}}$ siècle, avec l'implantation d'habitats pérennes (comme le hameau des Points à partir de 1467) ou éphémères.

La mise en perspective de la microrégion étudiée dans un cadre régional élargi permet donc de mesurer son apport à la connaissance des dynamiques de l'occupation du sol aux confins du Berry et du Nivernais. L'analyse microscalaire fournit l'expression locale de tendances décrites à partir d'autres sources à une échelle régionale et permet de nuancer ou de remettre en cause le caractère généralisé de ces tendances. L'approche micro-scalaire apparaît donc complémentaire de l'approche régionale sur ces questions : elle permet un élargissement de l'éventail des possibles, « une modulation locale de la grande histoire ${ }^{41} »$.

40. Michaud-FrejaVILLE 1997, p. 530-534.

41. REVEL 1989, p. XXI. 
Archéologie Médiévale, t.40 (2010), p.15-32

Bibliographie

BATARDY C., BuchSENSChÜTZ O., DUMASY F. (dir.)

2001, Le Berry antique - Atlas 2000, $21^{\mathrm{e}}$ Supplément à la Revue Archéologique du Centre de la France, Tours.

BERTONCELLO F. et NUNINGER L.

2004, «From archaeological sherds to qualitative information for settlement pattern studies», Actes du colloque CAA 2004 «Beyond the artifact - Digital interpretation of the past», Prato, Italy, 2004, http://halshs.archivesouvertes.fr/docs/00/07/73/61/PDF/bertoncello-nuninger_caa2004.pdf

BINTLIFF J. et SNODGRASS A.

1988, «Off-site potery distributions: A regional and interregional perspective», Current Anthropology, 29, p. 506-513.

BUHOT DE KERSERS A.

1892, Histoire statistique et monumentale du département du Cher, t.VI, Paris.

CHOUQueR G. (dir.)

1996a, Les formes du paysage t.1 - Études sur les parcellaires, Paris, Errance.

1996b, Les formes du paysage t. 2 - Archéologie des parcellaires, Paris, Errance.

1997, Les formes du paysage t.3- L'analyse des systèmes spatiaux, Paris, Errance.

DEVAILly G.

1973, Le Berry du X siècle au milieu du XIII siècle : étude politique, religieuse, sociale et économique, Paris, EPHE-Mouton.

FAURE-BOUCHARLAT E. (dir.)

2001, Vivre à la campagne au Moyen Âge, Lyon, DARA.

FERDIERE A., RIALLAND Y.

1994, «La prospection archéologique systématique sur le tracé de l'autoroute A71 (section Bourges-sud du Cher) », Revue archéologique du Centre de la France, 33, p. 7-86.

1995, «La prospection archéologique systématique sur le tracé de l'autoroute A71 (section Bourges-sud du Cher) », Revue archéologique du Centre de la France, 34, p. 5-87.

1996, «La prospection archéologique systématique sur le tracé de l'autoroute A71 (section Bourges-sud du Cher) », Revue archéologique du Centre de la France, 35 , p. 5-65.

FOARD G.

1978, «Systematic fieldwalking and the investigation of saxon settlement in Northamptonshire», World Archaeology, 9, p. 357-374.

GAFFNEY C. F., GAFFNEY V. L.

1988, «Some quantitative approaches to site territory and land use from the surface 
record», dans Bintliff J. L., Davidson D. A., G. GRANT E. (dir.), Conceptual issues in environmental archaeology, Édimbourg, Edinburgh University Press, p. 82-90.

GALLANT T. W.

1986, «'Background noise' and site definition: a contribution to survey methodology», Journal of Field Archaeology, 13, p. 403-418.

HUSI P. (dir.)

2003, La céramique médiévale et moderne du Centre-Ouest de la France (XI ${ }^{e}$-XVII siècle). Chrono-typologie de la céramique et approvisionnement de la Loire moyenne, Tours, FERACF.

JONES R.

2004, «Signatures in the soil: The use of pottery in manure scatters in the identification of medieval arable farming regimes», The Archaeological Journal, 161, 159-188.

LORREN C. et PERIN P. (dir.)

1995, L'habitat rural du haut Moyen Âge (France, Pays-Bas, Danemark et Grande Bretagne), 14e Journées Internationales d'Archéologie Mérovingienne, Guiry-enVexin et Paris, 4-8 février 1993, Rouen, Association Française d'Archéologie Mérovingienne.

MichAUD-FREJAVILLE F.

1997, «Économie et vie rurales en Berry à la fin du Moyen Âge », thèse de doctorat, Université de Paris I, Paris, dactyl.

\section{NUNINGER L.}

2002, «Peuplement et territoires protohistoriques du VIII ${ }^{\mathrm{e}}$ au $\mathrm{I}^{\mathrm{er}}$ s. av. J.-C. en Languedoc oriental (Gard, Hérault) », thèse de doctorat, Université de FrancheComté, Besançon, dactyl.

\section{PARODI A.}

1992, «La plaine du Languedoc oriental au haut Moyen-Age (IV $-X I^{\mathrm{e}}$ s.), Textes et archéologie de l'espace rural », thèse de doctorat, Université de Paris I, Paris, dactyl.

\section{POIRIER N.}

2006a, «Des plans terriers au cadastre ancien : Mesurer l'évolution de l'occupation du sol grâce au SIG», Le médiéviste et l'ordinateur, 44, http://lemo.irht.cnrs.fr/44/plans-terriers.htm.

2006b, «Du temps long au temps court : modéliser l'évolution spatiale de l'exploitation du sol», Actes du colloque Interactions Natures-Sociétés, La Baule, 3-5 mai 2006, UMR 6554 LETG, http://letg.univ-nantes.fr/COLLOQUE/pdf/PO_POIRIER.pdf.

2006c, «Statistiques spatiales et modélisation de l'exploitation du sol : variations d'échelles temporelles», Actes du colloque 1e rencontres de doctorants organisées par le RTP MODYS, Lyon, 8 et 9 novembre 2006, http://www.univtours.fr/isa/modys/download/rd06_poirier.pdf. 
Archéologie Médiévale, t.40 (2010), p.15-32

2007, «Un espace rural en Berry dans la longue durée : expérience de micro-analyse des dynamiques spatio-temporelles du paysage et du peuplement dans la région de Sancergues (Cher) », Thèse de doctorat, Université François-Rabelais, Tours, http://tel.archives-ouvertes.fr/tel-00212332/fr/.

2010, Un espace rural à la loupe - Paysages, peuplement et territoires en Berry de la préhistoire à nos jours, Tours, Presses Universitaires François-Rabelais.

POIROT A.

1998, «Neuvy-le-Roi (Indre-et-Loire), depuis ses origines jusqu'au XIX ${ }^{\mathrm{e}}$ siècle», Revue Archéologique du Centre de la France, 37, p. 139-178.

REVEL J.

1989, «L'histoire au ras du sol», dans LEVI G., Le pouvoir au village - Histoire d'un exorciste dans le Piémont du XVIIe siècle, Paris, Gallimard, p. I-XXXIII.

VAN DER LEEUW S, FAVORY F. et FICHES J.-L. (dir.)

2003, Archéologie et systèmes socio-environnementaux. Études multiscalaires sur la vallée du Rhône dans le programme ARCHAEOMEDES, Paris, CNRS.

WIEDERHOLD W. (dir.)

1910, Papsturkunden in Frankreich - V, Berry, Bourbonnais, Nivernais und Auxerrois, Berlin, Weidmann.

WILKINSON T.J.

1982, «The definition of ancient manured zones by means of extensive sherd-sampling techniques», Journal of Field Archaeology, 9, p. 323-333.

1989, «Extensive sherd scatters and land-use intensity: some recent results», Journal of Field Archaeology, 16, p. 31-46.

ZADORA-RIO É.

1987, «Archéologie du peuplement : la genèse d'un terroir communal», Archéologie Médiévale, t. 17, p. 7-65. 